\title{
Design and research of high precision nonmagnetic biaxial rotary table
}

\author{
Cui Wang ${ }^{1, \text { a }}$, Yong Zhang ${ }^{1, b}$, Ling Gao ${ }^{1, c}$, Zhibiao Ye ${ }^{1, d}$ \\ ${ }^{1}$ Department of Civil Engineering, Jiujiang Vocational and Technical College, No.1188 Ten-mile \\ Avenue, Jiujiang, Jiangxi Province, China, 332007 \\ awangcui19850608@126.com, b39431034@qq.com, cjgm8@163.com, dyezhibiao2000@163.com
}

Keywords: Biaxial rotary table, Nonmagnetic, High precision, Design and research

Abstract: Rotary tables are important machine tools in fields of angle measurement and attitude simulation. Nonmagnetic rotary table with two axes is designed and researched instead of traditional magnetic and electromagnetic measuring and control systems in this paper. T-U pattern is adopted in structural design of mechanical table, which is connected with measuring chassis by electric cable. Dynamic measurement of angular position is achieved from response inductosyn which provides favorable basis for the set of the equipment. Results of experiments show that, magnetic distortion measured of the developed nonmagnetic biaxial rotary table is $3.5 \mathrm{nT}$ to guarantee extremely weak magnetic field in the working area and angle test accuracy is less than 3 arc-second, which is among the front in nonmagnetic rotary table fields.

\section{Introduction}

As the transformation of warfare situation and conception brought by $3^{\text {th }}$ strategic nuclear missile, tactical missiles have greatly raised requirement in measurement method and precision. To meet the polydirectional demands of multiple goals and tasks, geomagnetic disturbance in the process of accurate acquiring flight attitude parameters shall be solved effectively. As a matter of fact, lots of researchers had already studied magnetic measuring and attitude control systems [1-5]. Further more, Nona etal [6] made efforts on hybrid attitude controller consisting of electromagnetic torque rods and an active fluid ring. Many manufacturers such as Koma, Yuasa and Aerotech have designed advanced magnetic motion simulators (Figure 1), sensor test and control systems [7]. Also, three-axis attitude controller was researched through magnetic modes and magnetorquers [8-10]. But as for nonmagnetic attitude measuring, little has been found in the present literature, not to mention design method and experiment on situ.

According to past researches and experiments, various attitude control systems for different spacecrafts had been developed such as fuzzy proportion integration differentiation control and periodic linear systems by bounded controls etc [11-15]. In addition, magnetic attitude control systems using air-bearing-based attitude control simulator or without initial detumbling were studied in recent years $[16,17]$. Since different magnetic field will cause large interference in the test area which results in great deviation in the attitude measuring, to meet the higher requirement of modern air vehicles as well, design and research on a system of attitude simulation equipment with high precision in measuring flight attitude which is consists of nonmagnetic materials becomes more and more crucial in the spacecrafts manufacture process.

Based on the conceptual design method of magnetic or electromagnetic guided rotary table [18.19], nonmagnetic biaxial rotary table is developed and tested in this paper. As for error analysis, several calibration strategies [20-23] were observed before starting the measuring experiments so as to improve the accuracy. The developed biaxial nonmagnetic rotary table called 2FHT300C, the whole designing and experimenting process is stated as follows. 


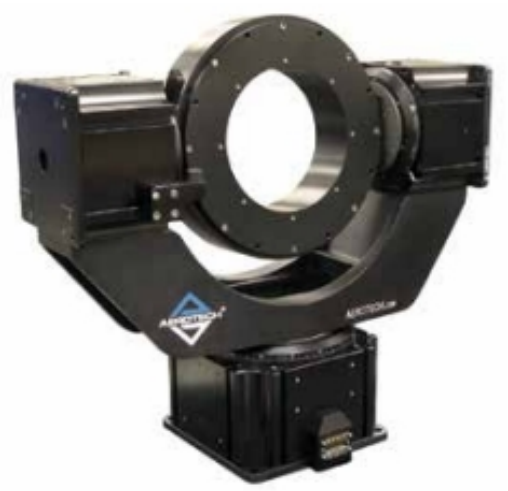

Figure 1 Typical biaxial air-bearing motion simulator by Aerotech

\section{Design and Research}

2FHT300C biaxial manual nonmagnetic rotary table is composed by mechanical table and measuring chassis. T-U frame is adopted in table structure designing with elevation axis in the inner race and azimuth axis in the outer race respectively (Figure 2). Dynamic measurement of angular position is realized by utilizing synchronous sensors. Rotations of shafts are driven manually by turbine and worm at two different levels.

\section{Mechanical Table}

As is shown in Figure 2, in the mechanical tale, both of azimuth and elevation axes are supported by precise ceramic mechanical axle bearing where synchronous sensors are installed as the elements of angle measuring. $U$ shape erecting fixture contains clump weight whose resolution ratio is within the static friction torque limits. When the specimen and fixture are installed in the plane, balance of azimuth and elevation axes can be guaranteed by additional set up of clump weight. M8 threaded holes are distributed uniformly in separate diameters to assure different test specimen to be correctly installed.
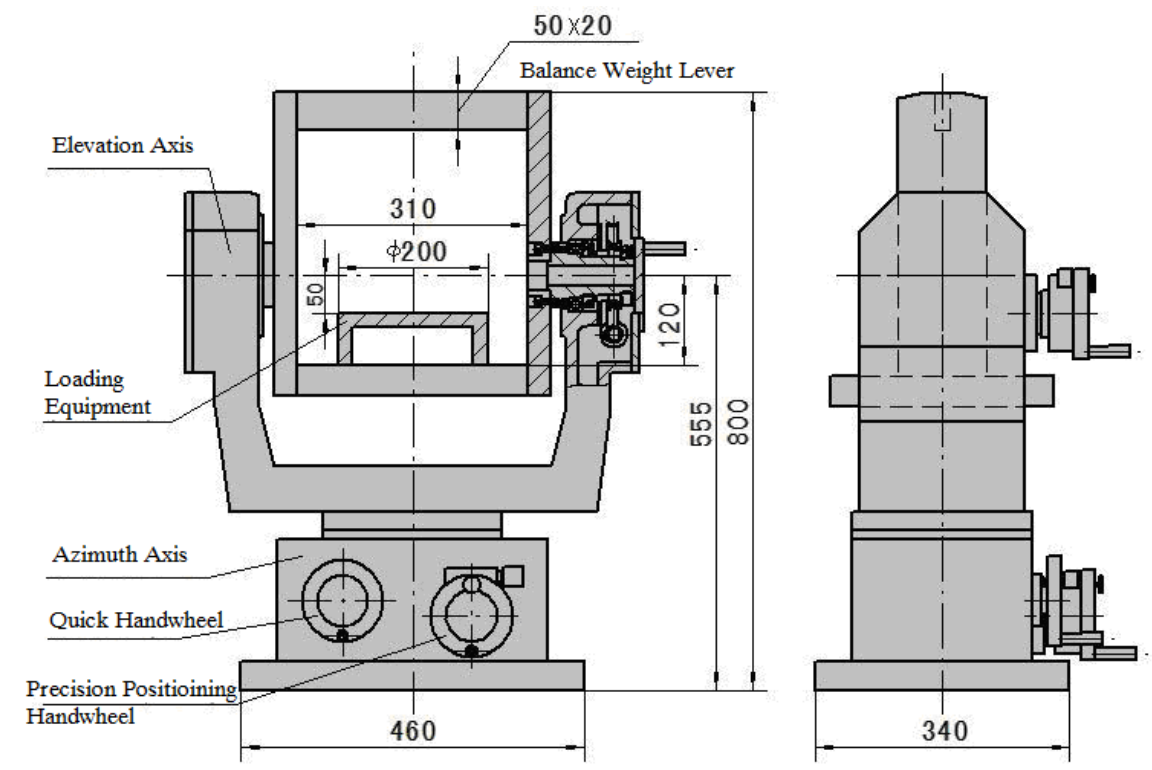

Figure 2 Blue print of 2FHT300C biaxial manual nonmagnetic rotary table

The installation plane of $2 \mathrm{FHT} 300 \mathrm{C}$ is $\Phi 200 \mathrm{~mm}$, end beat $0.02 \mathrm{~mm}$ and flatness is $0.02 \mathrm{~mm}$. The load limits of test specimen is $10 \mathrm{~kg}$ and the size is smaller than $\Phi 200^{*} 300$. Both azimuth axis and elevation axis have continuous angle ranges and the angle rotation error is less than 5". Non-perpendicularity of azimuth and elevation axis is less than 5" while the intersection of azimuth and elevation axis is no greater than $\Phi 0.2 \mathrm{~mm}$ sphere.

As for angle measurement, angular position resolution ratio is $0.0001^{\circ}$, whose angular position precision is $\pm 5^{\prime \prime}$, angular position repetitiveness \pm 1 " and angular rate ranges from $0.01 \%$ s to $360 \%$ s. 
Two axis angles are displayed in Nixie tube as $\mathrm{xxx} . \mathrm{xxxx}^{\circ} .12$ subscriber loops were designed in 2FHT300C. The rated current is $2 \mathrm{~A}$ with an temperature conditions of $20^{\circ} \mathrm{C} \pm 8^{\circ} \mathrm{C}$ and electric resistance of table landing less than $2 \Omega$.

\section{Measuring Chassis}

Figure 3 is the front panel of measuring chassis, which is connected with mechanical table by electric cable so as to conduct signals from synchronous sensors. A computer is linked to the chassis by RS-232 serial ports with Baud rate of $115.2 \mathrm{kbps}$ which enables real-time recording, tracking measuring, digital display, saving and outputting of angle data from the azimuth and elevation axis.

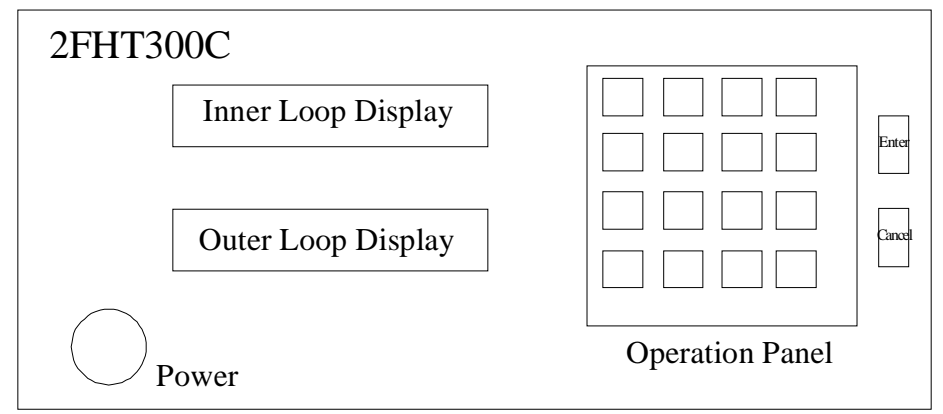

Figure 3 Front panel of measuring chassis in 2FHT300C

\section{Nonmagnetic Materials and method}

In order to apply the developing apparatus in magnetic metrology and calibration, further more, detection of magnetic navigation devices, utilizing nonmagnetic materials which do not conduct magnetism and relatively hard to be magnetized becomes the basic and core issue in the process of developing nonmagnetic attitude simulator with high precision. The main problems to be solved are:

1) Overall plan of nonmagnetic attitude simulator;

2) According to the nonmagnetic requirement, special controlling scheme shall be designed in order to avoid electromagnetism disturbance produced by motor drive, test accuracy shall not be influenced at the same time.

3) Magnetic distortion shall be less than $7 \mathrm{nT}$ to guarantee the characteristic of nonmagnetic simulator.

To settle the issues raised above, ZALSi9mg aluminum casting alloy is utilized to shape and build support frame and foundation. After casting, non magnetic detection is conducted and further machining shall be carried out only when detection is accordingly qualified.

Supporting shafts installed in the ceramic bearing is composed by 2A12 duralumin and is driven by turbine and worm which is H62 brass made because shafts are the key components and their mechanical precision and system performance is strongly affected by structural design and materials, such as bearing type, brace design and friction torque.

Technique of angular contact ball bearing and proper pretightening force is applied in both ends of azimuth and elevation axes which guarantee the accurate axial and radial positioning and tilting precision as well. Synchronous sensors are fixed along the coaxial and precision conductive ring is installed in the center-hollowed axis. Support frame cast by high strength aluminum alloy, coupling part of azimuth and elevation axes, is designed as sealed hollow structure with stiffened plate to achieve higher strength and stiffness.

Other auxiliary machinery parts are manufactured by qualified aluminum alloy while standard fastens by brass.

\section{Angle measuring system}

Synchronous sensors are applied in the angle measuring system utilizing pumping in single phase and discrimination in two phases which is composed by pumping module, operation module and power-supply module. In detail, pumping module provides pumping signals of synchronous sensor, and operation module collects the signals to do angular position operation so as to offer angular position data to controlling and management system (Figure 4). 


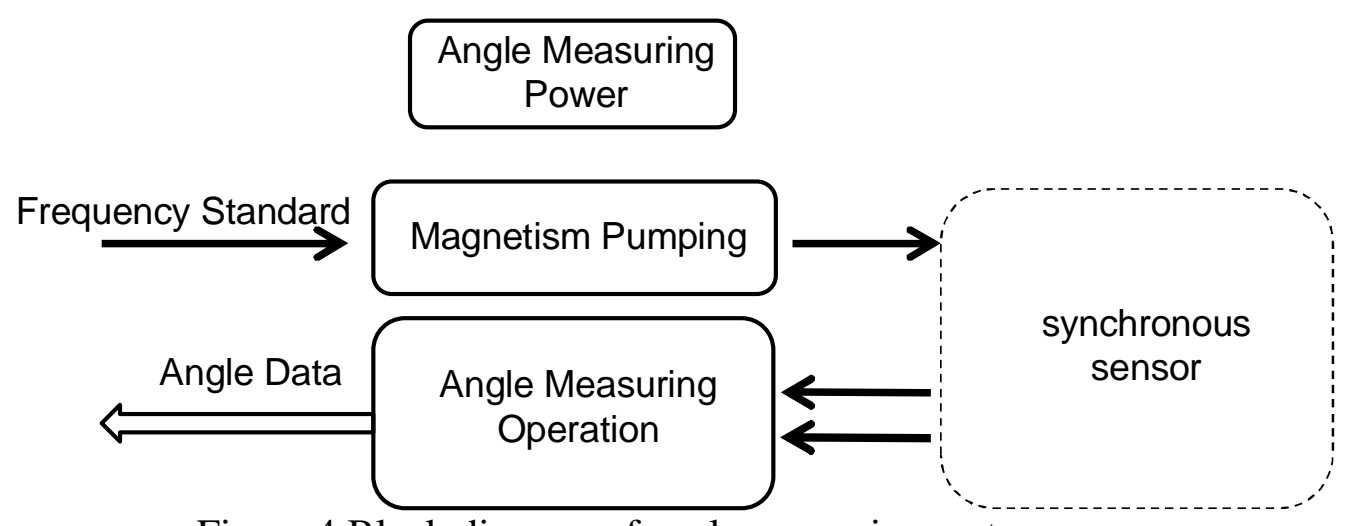

Figure 4 Block diagram of angle measuring system

Sine pumping signal output by excitatory power transmit to rotor continuous winding of synchronous sensors and rotary transformer while two-phase winding signals of stator transmit to angle measuring operation module.

$$
\begin{aligned}
& \theta_{S}(t)=V_{S}(t) \cdot \sin \theta \cdot \sin \omega t \\
& \theta_{C}(t)=V_{c}(t) \cdot \cos \theta \cdot \sin \omega t
\end{aligned}
$$

As long as the fluctuate amplitude of $V_{S}(t)$ and $V_{C}(t)$ in Equation 1 and 2 is within certain limits, frequency of fluctuation is far less than that of measurement sampling in angle measuring system. Precise $\sin \theta$ and $\cos \theta$ function of sensor electric angle can be obtained through sampling filter. Further, $\theta$ can be calculated by functional transformation and finally angle data can be obtained after tracking measurement (Figure 5).

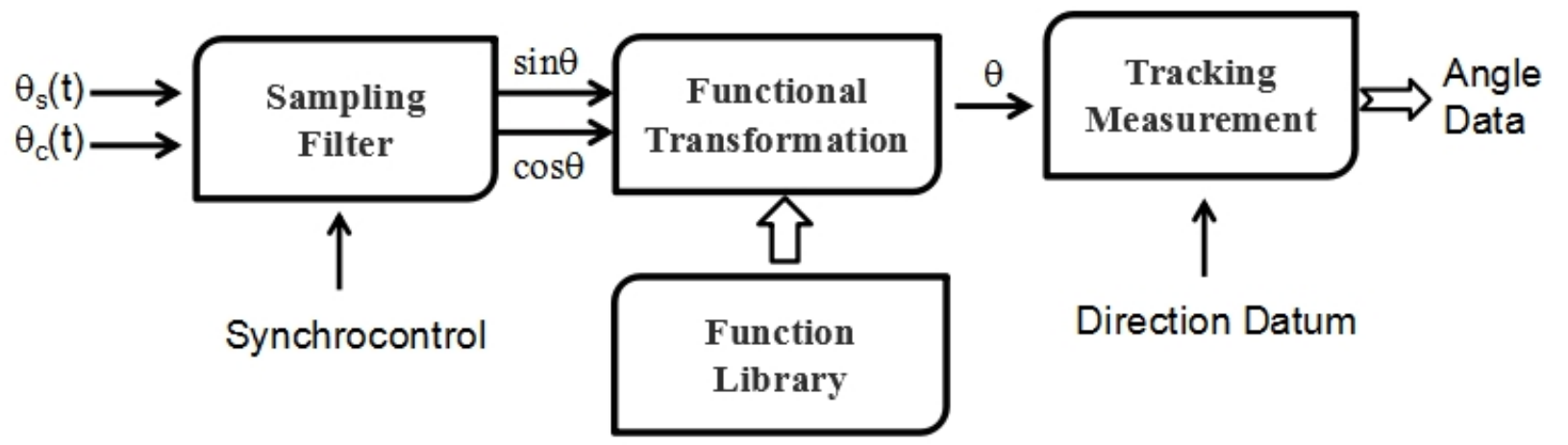

Figure 5 Principle of operation module in angle measuring system

\section{Results}

\section{Magnetic field measuring results}

One measuring task of the rotary table is the magnetism of using materials which belongs to the weak magnetic field and the other is the distortion of the uniform stable magnetic field which belongs to the normal measurement range. Both measuring method require high resolution ratio at a level of $0.1 \mathrm{nT}$. According to Table 1, methods of magnetic resonance, Hall effect and electromagnetic induction are suitable for normal magnetic field measurement, magneto-optical effect method for super strong magnetic filed, while flux gate, optical pump and superconductor quantum interference device method for weak magnetic field. Considering additional requirement for precision, flux gate are applied in magnetism measurement of the project. 
Table 1 Engineering magnetic field measurement [24]

\begin{tabular}{ccccc}
\hline Method & Suitable magnetic field & Scope $(\mathrm{Gs})$ & Precision & Features \\
\hline Magnetic resonance & uniform & $1-2 \times 10^{4}$ & $10^{-5}-10^{-7}$ & high precision \\
Hall effect & DC or pulse & $1-10^{5}$ & $10^{-2}-10^{-4}$ & good linearity small probe \\
Electronmagnetic induction & DC or AC & $0-$ & $10^{-2}-10^{-4}$ & simply method wide range \\
Flux gate & weak & $\leqq 100$ & $10^{-3}$ & high sensitivity light weight \\
Optical pump & weak & $10^{-3}-10^{-6}$ & $10^{-7}$ & high sensitivity, readable \\
Magneto-optical effect & strong pulse and DC & $>10^{5}$ & $10^{-2}$ & Safe and reliable \\
Magnetic film & weak mainly in military & $\leqq 10^{-4}$ & $10^{-5}$ & low noisesimple structure \\
Superconductor quantum & slight & $10^{-9}-10^{-3}$ & $10^{-10}$ & high resolution ratio and price \\
\hline
\end{tabular}

Before the rotary table installed in place, magnetic field intensity are measured at 8 desired points in work areas by admeasuring apparatus with customized nonmetal localized probe in three coordinate directions to get $H_{x 1} \ldots \ldots . H_{x 8} 、 H_{y 1} \ldots . . . H_{y 8} 、 H_{z 1} \ldots \ldots . H_{z 8}$ respectively. After the installation, $H_{x 1}^{\prime} \ldots . . . H_{x 8}^{\prime} 、 H_{y 1}^{\prime} \ldots \ldots . H_{y 8}^{\prime} 、 H_{z 1}^{\prime} \ldots . . . H_{z 8}^{\prime}$ are measured at the same points where measuring probes are localized at top of the table. Variation of magnetic field intensity can be calculated as $\Delta H_{x i}=H_{x i}^{\prime}-H_{x i}, \Delta H_{y i}=H_{y i}^{\prime}-H_{y i}, \Delta H_{z i}=H_{z i}^{\prime}-H_{z i}$ where $i=1,2, \ldots 8 \cdot\left\{\left|\Delta H_{j i}\right|\right\}_{\max }, j=x, y, z$ is the maximum value of the computing result, and the observed magnetic field is $3.5 \times 10^{-3} \mathrm{Gs}$, namely $3.5 \mathrm{nT}$.

\section{Angle measuring results}

Compared to ordinary mechanical methods, angular surveying obtains higher accuracy by optical means and is apt to subdivision. Till now, optical angle measurement methods mainly contain optical dividing head, polygonal prism, circular grating, circular ring laser, laser interference, etc. As for 2FHT300C, polygonal prism measuring method was chosen to research on the precision because of its high accuracy. Polygon is a regularprism whose cardinal plane is the bottom. Each working face can form a plurality of angle values similar to multi angle gauge. Polygon is usually used in conjunction with an autocollimator which collimate and fix position of working faces in polygon by its optic axis.

According to GJB1801 [25], 23-surface polygon was used in the research of angle measurement. When the axis angle showed 0 , the initial position was determined and record initial reading of light pipe $\mathrm{c} 1$. Based on the digital display, turn a degree of $15.6522^{\circ}$ once at a time, and record the corresponding indication of the light pipe $\mathrm{c} 2 \ldots \mathrm{c} 23$.

$$
e_{a i}=c_{i}-c_{1}-\Delta_{i}
$$

where $e_{a i}$ is the angle error of each testing point and $\Delta_{i}$ is corrected value of corresponding surface. Among the computing results, positive maximum error $e_{0}^{+}$and negative maximum error $e_{0}^{-}$ were obtained. Assumed that $+U_{a}$ equals $e_{0}^{+}$and $-U_{a}$ equals $e_{0}^{-}$, then accuracy of angle position measurement are $+U_{a},-U_{a}$.

Both elevation and azimuth axes were measured by polygonal prism method, the results were showed in Figure 6 and Figure 7. From the figures, the positive and negative maximum errors (Solid lines) are $R=2.8^{\prime \prime}, r=1.3^{\prime \prime} ; R, r \leq 3^{\prime \prime}$. Further more, as for $84.13 \%$ guaranteed rate, the sums of average error and standard deviation of both axes (Dotted lines) are calculated as $R^{\prime}=2.22^{\prime \prime}, r^{\prime}=0.75^{\prime \prime} ; R^{\prime}, r^{\prime} \leq 2.3^{\prime \prime}$ 


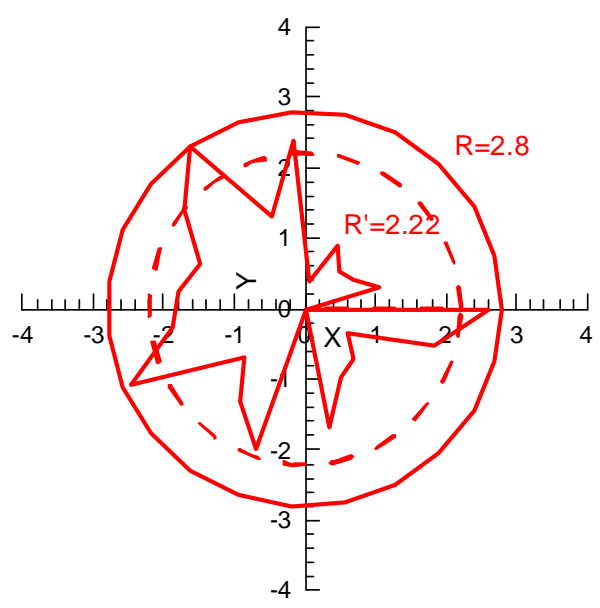

Figure 6 Errors of angle measuring in elevation axis

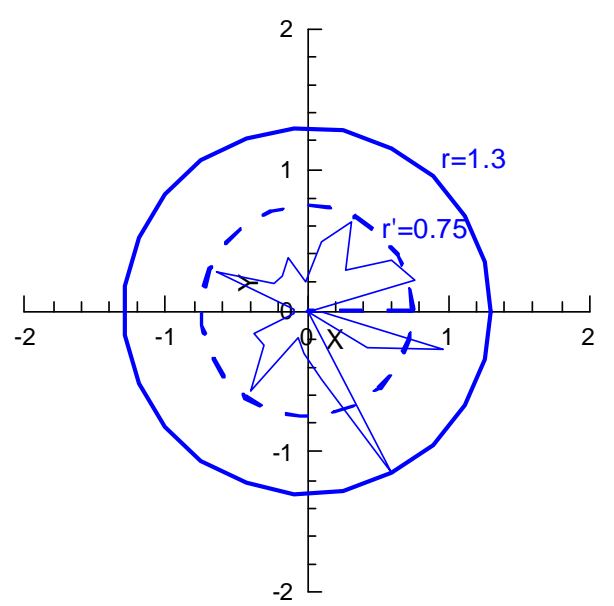

Figure 7 Errors of angle measuring in azimuth axis

\section{Conclusions}

Based on the nonmagnetic and high precision design concept and measuring methods, nonmagnetic biaxial rotary table was designed and researched, conclusions are as follows:

1) Overall plan of nonmagnetic attitude simulator is accomplished. T-U pattern is adopted in structural design of mechanical table, which is connected with measuring chassis by electric cable. Dynamic measurement of angular position is achieved from response inductosyn which provides favorable basis for the set of the equipment.

2) By applying nonmagnetic materials and technology, magnetic distortion of the developed nonmagnetic biaxial rotary table is $3.5 \mathrm{nT}$, which is less than $7 \mathrm{nT}$ to guarantee extremely weak magnetic field in the working zone.

3) Angle test accuracy applying polygon prism method is less than 3 arc-second, which is among the front in the nonmagnetic rotary table fields.

\section{Acknowledgments}

The active support received from Scientific and Technological Research Program of Jiangxi Province (Project No.GJJ151373), during the preparation of this paper is gratefully acknowledged.

\section{References}

[1] M. Yu. Ovchinnikov, D.S. Roldugin, V.I. Penkov. Asymptotic study of a complete magnetic attitude control cycle providing a single-axis orientation [J]. Acta Astronautica, Volume 77, pp48-60, 2012

[2] A. Craig Stickler, K.T. Alfreind. Elementary magnetic attitude control system [J]. Journal of Spacecraft, Vol.13, No.5, pp282-287, 1975

[3] Rafal Wisniewski, Mogens Blanke. Fully magnetic attitude control for spacecraft subject to gravity gradient [J]. Automatica, Volume 35, pp1201-1214, 1999

[4] Enrico Silani, Marco Lovera. Magnetic spacecraft attitude control: a survey and some new results [J]. Control Engineering Practice, Volume. 13, pp357-371, 2005

[5] M. Lovera, A. Astolfi. Spacecraft attitude control using magnetic actuators [J]. Automatica, Volume 40, pp1405-1414, 2004

[6] Nona A. Nobari, Arun K. Misra. A hybrid attitude controller consisting of electromagnetic torque rods and an active fluid ring [J]. Acta Astronautica, Volume 94, pp470-479, 2014

[7] Advanced motion systems for aerospace, defense and national security, www.aerotech.com

[8] M.Yu. Ovchinnikov, D.S. Roldugin, V.I. Penkov etc. Fully magnetic sliding mode control for acquiring three-axis attitude [J]. Acta Astronautica, Volume 121, pp59-62, 2016 
[9] Fabio Celani. Robust three-axis attitude stabilization for inertial pointing spacecraft using magnetorques [J]. Acta Astronautica, Volume 107, pp87-96, 2015

[10] M.Yu. Ovchinnikov, D.S. Roldugin, V.I. Penkov. Three-axis active magnetic attitude control asymptotical study [J]. Acta Astronautica, Volume 110, pp279-286, 2015

[11] Marco Lovera, Alessandro Astolfi. Global magnetic attitude control of spacecraft in the presence of gravity gradient [C]. IEEE transactions on aerospace and electronic systems, Vol.42, No.3, 2006

[12]Cui Wang. Research and simulation of vehicle lateral stability control based on fuzzy PID control [D]. Changsha University of Science and Technology, 2009

[13] Daniel Calvo, Taisir Aviles, Vitoria Lapuerta, etc. Fuzzy attitude control for a nanosatellite in low earth orbit [J]. Expert Systems With Applications, Volume 58, pp102-118, 2016

[14]Bin Zhou. Global stabilization of periodic linear systems by bounded controls with applications to spacecraft magnetic attitude control [J]

[15] Mohammad Abdelrahman, Sang-Young Park. Integrated attitude determination and control system via magnetic measurements and actuation [J]. Acta Astronautica, Volume 69, pp168-185, 2011

[16]H.S. Ousaloo, M.T. Nodeh, R. Mehrabian. Verification of spin magnetic attitude control system using air-bearing-based attitude control simulator [J]. Acta Astronautica, article in press, 2016

[17]D.S. Roldugin, P. Testani. Spin-stabilized satellite magnetic attitude control scheme without initial detumbling [J]. Acta Astronautica, Volume 94, pp446-454, 2014

[18]Farhad Bayat, Hossein Bolandi, Ali A. Jalali. A heuristic design method for attitude stabilization of magnetic actuated satellites [J]. Acta Astronautica, Volume 65, pp1813-1825, 2009

[19]B. Denkena, D. Dahlmann, F. Floeter, etc. Conceptual design for electromagnetic guided rotary table in machine tools [C]. $5^{\text {th }}$ Machining Innovations Conference, pp80-85, 2014

[20] Wataru Kokuyama, Tsukasa Watanabe, Hideaki Nozato, etc. Angular velocity calibration system with a self-calibratable rotary encoder [J]. Measurement, Volume 82, pp246-253, 2016

[21] Velenosi Alessandro, Campatelli Gianni, Scippa Antonio. Axis geometrical errors analysis through a performance test to evaluate kinematic error in a five axis tilting-rotary table machine tool [J]. Precision Engineering, Volume 39, pp224-233, 2015

[22] Shitu Luo, Hongfeng Pang, Ji Li, etc. Calibration strategy and generality test of three-axis magnetometers [J]. Measurement, Volume 46, pp3918-3923, 2013

[23]I. Grybas, R. Bansevicius, V. Jurenas, etc. Ultrasonic standing waves-driven high resolution rotary table [J]. Precision Engineering, Volume 45, pp396-402, 2016

[24]Zhouli Wu. Research on automatic non-magnetic three-axis rotary table [D]. Huazhong University of Science and Technology, 2007

[25] GJB1801-93 Testing methods of major performance for test equipments of inertial technology [S]. National military standard of the people's Republic of China 